\title{
Review
}

\section{Xenobiotic metabolism in the zebrafish: a review of the spatiotemporal distribution, modulation and activity of Cytochrome P450 families 1 to 3}

\author{
Moayad Saad, Kate Cavanaugh, Evy Verbueken, Casper Pype, Christophe Casteleyn, \\ Chris Van Ginneken and Steven Van Cruchten
}

\author{
Applied Veterinary Morphology, Department of Veterinary Sciences, Faculty of Pharmaceutical, \\ Biomedical and Veterinary Sciences, University of Antwerp Universiteitsplein 1, B-2640 Wilrijk, Belgium
}

(Received June 4, 2015; Accepted November 12, 2015)

\begin{abstract}
The zebrafish (Danio rerio) has been increasingly explored in pharmaceutical research as a promising alternative model for toxicological screens. This necessitates a thorough knowledge on the biotransformation processes for a correct interpretation of pharmacological and toxicological data. Physiologically, cytochrome P450 (CYP) enzymes, specifically CYP families 1-3, play a pivotal role in drug metabolism. And yet, information regarding activity of CYP, its isoforms, and conjugation enzymes in zebrafish is either scarce or conflicting. To account for this discrepancy, the available spatiotemporal, modulation and activity data on zebrafish CYP 1-3 families are reviewed in this paper and compared with human CYP data. The CYP genetic features and synteny are well characterized, as is their expression in different organ systems. Moreover, several substrates metabolized by humans also show metabolism in zebrafish, with other CYP isoforms possibly involved. Altogether, the five CYP1 members, 41 CYP2 members and five CYP3 members in zebrafish show distinct evolutionary and orthological similarities with humans.
\end{abstract}

Key words: Zebrafish, Cytochrome P450, Biotransformation, Xenobiotics, Ontogeny, Synteny

\section{INTRODUCTION}

The zebrafish (Danio rerio) is a vertebrate organism that has become ubiquitous in modeling development and toxicology. This laboratory animal offers the power of whole-animal investigations paired with the convenience of cell culture techniques that are cost- and time-effective, as well as requiring minimal infrastructure. From a morphological standpoint, the zebrafish is a convenient model compared to its higher vertebrate counterparts. The transparency of the embryo, in particular, affords simple, non-invasive visual analysis of developmental processes in vivo. Marked by high fecundity, the zebrafish allows for large sample sizes with fast organismal development and maturation. Therefore, this organism has become a highly desired alternative model for early selection of compounds during the drug development process. Since the toxic effects of compounds depend on the achieved exposure within the organism, a thorough knowledge of the biotransformation processes is necessary for proper safety assessment. However, biotransformation capacity in zebrafish adults and embryos still needs more elucidation (Beker van Woudenberg et al., 2013; Brannen et al., 2010; Weigt et al., 2012)

Drug metabolism is largely achieved by an oxidative reaction catalyzed through cytochrome P450 enzymes (CYPs). Originally found in rat liver microsomes, CYPs are in fact present in all eukaryotic organisms (such as animals, plants, and fungi) and even in some prokaryotes. CYPs are divided into families (CYP1, 2, 3, etc.) when they exhibit an amino acid identity of more than $40 \%$ and into subfamilies (CYP1A, 1B, 1C, etc.) when more than 55\% (Brown et al., 2008). Using the Ensembl genome browser (release 82), which incorporates the most recent annotations on the zebrafish genome from the Genome Reference Consortium (GRCz10), a total of 86 CYP genes that fall into 17 categories of $C Y P$ gene families were found (Table 1) (Genome Reference Consortium, 2015). Of these CYP families, CYP 1-3 are the main xenobiotic metabolizing enzymes responsible for drug

Correspondence: Steven Van Cruchten (E-mail: steven.vancruchten@uantwerpen.be) 
M. Saad et al.

Table 1. Zebrafish CYP families 1, 2 and 3.

\begin{tabular}{|c|c|c|c|c|c|c|c|c|}
\hline \multirow{2}{*}{$\begin{array}{l}\text { CYP1s } \\
\text { CYP1A }\end{array}$} & \multicolumn{7}{|c|}{ CYP2s } & \multirow{2}{*}{$\frac{\text { CYP3s }}{\text { CYP3A65 }}$} \\
\hline & CYP2AA1 & CYP2AE1 & CYP2K6 & CYP2AD2 & CYP2R1 & CYP2X6 & CYP2Y3 & \\
\hline CYP1B1 & CYP2AA2 & & CYP2K8 & CYP2AD3 & CYP2U1 & CYP2X7 & & CYP3C1 \\
\hline CYP1C1 & CYP2AA3 & & CYP2K16 & CYP2AD6 & & CYP2X8 & & CYP3C2 \\
\hline CYP1C2 & CYP2AA4 & & CYP2K17 & CYP2J20 & & CYP2X9 & & CYP3C3 \\
\hline \multirow[t]{7}{*}{ CYP1D1 } & CYP2AA6 & & CYP2K18 & CYP2N13 & & CYP2X10.2* & & CYP3C4 \\
\hline & CYP2AA7 & & CYP2K19 & CYP2P6 & & CYP2X10.2** & & \\
\hline & CYP2AA8 & & CYP2K20 & CYP2P7 & & & & \\
\hline & CYP2AA9 & & CYP2K21 & CYP2P8 & & & & \\
\hline & CYP2AA11 & & CYP2K22 & CYP2P9 & & & & \\
\hline & CYP2AA12 & & CYP2K31 & CYP2P10 & & & & \\
\hline & & & & CYP2V1 & & & & \\
\hline
\end{tabular}

*ENSDARG00000068283, ** ENSDARG00000006501

and fatty acid metabolism, yet they display a wide variation in functions in vivo. It is important to note that of the 57 CYP enzymes encoded in the human genome, only around 15 are involved in xenobiotic metabolism and only five CYPs account for $95 \%$ of phase I metabolism of all marketed drugs (Guengerich, 2008).

In the zebrafish, the full complement of $C Y P$ genes and their developmental expression have been well documented previously (Goldstone et al., 2010), but data on tissue distribution, modulation and activity of CYPs, which are important factors in the metabolism and clearance of xenobiotics, are scattered. Indeed, many drugs increase CYP activity by inducing the biosynthesis of CYP isoenzymes through particular signaling pathways. As such, the metabolism and clearance of xenobiotics are also affected. In humans, constitutive androstane receptor (CAR), pregnane X-receptor (PXR) and aryl hydrocarbon receptor (AHR) are well known transcription factors controlling CYP induction (Waxman, 1999). Zebrafish only exhibit two of these three regulatory mechanisms, with $C A R$ being absent in zebrafish and teleost fish in general (Reschly and Krasowski, 2006). The nuclear hormone receptor PXR and CYP3A are induced in zebrafish with a similar mechanism as in man (Bresolin et al., 2005). However, the number of substrates that stimulate $P X R$ is more limited in zebrafish (Ekins et al., 2008). Regarding AHR, this cytosolic protein complex translocates to the nucleus after ligand binding and dimerizes with the aryl hydrocarbon receptor nuclear translocator (ARNT). This leads to a high DNA binding affinity of this complex to stimulate gene transcription of the CYP1A1 and other genes (Denison and Nagy, 2003). This AHR mechanism pathway is highly conserved across taxa, suggesting a phys- iological xenobiotic-independent function of the AHR (Hahn, 2002). Mammals including humans have only one functional AHR, whereas zebrafish AHR have multiple signaling members including AHR1a, AHR1b, AHR2, ARNT1, ARNT2 and two AHR repressors (Karchner et al., 2005). AHRs have affinity to a broad range of aromatic and halogenated chemicals including planar halogenated aromatic hydrocarbons ( $\mathrm{pHAH})$ and polycyclic aromatic hydrocarbons (PAH), which are both known as environmental contaminants (Otte et al., 2010).

The following review will consecutively discuss the available data on spatiotemporal distribution, modulation and activity of the CYP1, 2 and 3 families in zebrafish. These data will also be related to human CYP data when available (Table 2). The term CYP is fully capitalized in all cases except when referring to a specific gene, which is italicized, consistent with the nomenclature committee recommendations (Nelson, 2009).

\section{CYP1 FAMILY}

In zebrafish, the five known CYP1 isoforms are CYP1A, CYP1B1, CYP1C1, CYP1C2, and CYP1D1 (Goldstone et al., 2010). Except for CYP1D1, all of these CYP subfamilies are upregulated by AHR activation and induced by several compounds such as polychlorinated biphenyls (PCB), beta-naphthoflavone, benzo[a]pyrene (BaP), pesticides and 2,3,7,8-tetrachlorodibenzop-dioxin (TCDD) (Jönsson et al., 2007a, 2007b; Goldstone et al., 2009). Additionally, most CYP1 isoforms are induced by exposure to oxidative stress and ultraviolet (UV) radiation (Behrendt et al., 2010). Interestingly, pregnenolone (PN), which is a specific PXR agonist, also induces zebrafish 
CYPs in zebrafish

Table 2. Synteny between zebrafish and human CYPs.

\begin{tabular}{|c|c|c|c|}
\hline Family & Subfamily & Zebrafish CYPs & Human CYPs \\
\hline \multirow{4}{*}{ CYP1 } & A & $1 \mathrm{~A}$ & $1 \mathrm{~A} 1,2$ \\
\hline & $\mathrm{B}$ & 1B1 & 1B1 \\
\hline & $\mathrm{C}$ & $1 \mathrm{C} 1,2$ & - \\
\hline & $\mathrm{D}$ & 1D1 & Inactive CYP1D1 gene \\
\hline \multirow{11}{*}{ CYP2 } & AA & $2 \mathrm{AA}(1-4,6-9,11,12)$ & - \\
\hline & $\mathrm{AE}$ & 2AE1 & - \\
\hline & $\mathrm{C}$ & & $2 \mathrm{C}(8,9,18,19)$ \\
\hline & $\mathrm{D}$ & - & 2D6 \\
\hline & $\mathrm{E}$ & - & $2 \mathrm{E} 1$ \\
\hline & $\mathrm{K}$ & $2 \mathrm{~K} 6,2 \mathrm{~K} 8,2 \mathrm{~K} 16-22,2 \mathrm{~K} 31$ & 2W1 \\
\hline & $\mathrm{P}, \mathrm{AD}, \mathrm{V}, \mathrm{N}$ & $2 \mathrm{AD}(2,3,6), 2 \mathrm{~J} 20,2 \mathrm{~N} 13,2 \mathrm{P}(6,7,10), 2 \mathrm{~V} 1$ & $2 \mathrm{~J} 2$ \\
\hline & $\mathrm{U}$ & $2 \mathrm{U} 1$ & $2 \mathrm{U} 1$ \\
\hline & $\mathrm{R}$ & 2R1 & 2R1 \\
\hline & $X$ & $2 X(6-10)$ & - \\
\hline & $\mathrm{Y}$ & $2 \mathrm{Y} 3$ & 2(A6,A13,B6,F1,S1) \\
\hline \multirow{2}{*}{ CYP3 } & A & $3 \mathrm{~A} 65$ & Inactive CYP3A genes \\
\hline & $\mathrm{C}$ & $3 C(1-4)$ & $3(\mathrm{~A} 3, \mathrm{~A} 4, \mathrm{~A} 7)$ \\
\hline
\end{tabular}

CYP1A (Kubota et al., 2015).

In humans, the activity and/or the expression of these enzymes can be downregulated by several mechanisms. For example, $\alpha$-naphthoflavone (ANF) is a competitive inhibitor of CYP1A and at the same time a partial antagonist of AHR (Merchant et al., 1992; Miranda et al., 1998). L-fluoranthene (FL) lowers the CYP1A protein levels in vivo and inhibits non-competitively CYP1A in vitro, whereas piperonyl butoxide (PBO) forms a complex with the hem group leading to a nonselective inhibition of all CYPs including CYP1s (Franklin, 1977).

Humans and zebrafish show a broad spectrum of substrates metabolized by CYP1s. Many human CYP1, 2 and 3 probes are metabolized by zebrafish CYP1s. For instance, 7-ethoxyresorufin (ER), 7-methoxyresorufin (MR), 17 $\beta$-estradiol (E2), and benzo[a]pyrene (BaP) (human CYP1 probes) (Shimada et al., 1996), and 7-benzyloxyresorufin (BR) (CYP2 and 3 probe) (Niwa et al., 2003) are metabolized by zebrafish CYP1s (Scornaienchi et al., 2010a, 2010b). Surprisingly, 7-benzyloxy-4(trifluoromethyl) coumarin (BFC), which is a selective substrate for CYP3A in humans (Crespi and Stresser, 2000), is metabolized by zebrafish CYP1A more efficiently than zebrafish CYP3A65 (Scornaienchi et al., 2010b). Additionally, E2 which is metabolized by the human CYP1B1 is metabolized more effectively in zebrafish by
CYP1C1 and CYP1A rather than CYP1B1 (Scornaienchi et al., 2010a).

\section{CYP1A}

In adult zebrafish, the highest basal expression of CYP1A mRNA can be found in liver and gut tissue. Compared to other CYP1s, CYP1A mRNA levels are the highest in organs of the abdominal cavity and the heart (Jönsson et al., 2007b), with expression mainly in the cardiovascular system during the embryonic development (Otte et al., 2010).

In this respect, CYP1A mRNA expression is low during early development and increases drastically after hatching around 72 hours post-fertilization (hpf) (Goldstone et al., 2009). Because of this gap in expression, Mattingly and Toscano (2001) suggested that a posttranscriptional silencing mechanism exists in embryos until $72 \mathrm{hpf}$. This silencing is believed to be a protective mechanism in early developmental stages against cardiovascular abnormalities caused by the CYP1A biotransformation of some environmental pollutants (Mattingly and Toscano, 2001). In contrast, Otte et al. (2010) used 7-ethoxyresorufin-O-deethylase (EROD) assays for CYP1A activity and found peak levels at $8 \mathrm{hpf}$. This activity reached the lowest level at $36 \mathrm{hpf}$ and then increased until another peak at $104 \mathrm{hpf}$ (Otte et al., 
2010).

Planar halogenated aromatic hydrocarbons (pHAHs), like TCDD, have been proven to induce CYP1A and cause vascular abnormalities in early zebrafish developmental stages (Andreasen et al., 2002; Henry et al., 1997). The localization of AHR2 and ARNT2 in parallel to CYP1A in the vasculature of early embryos, suggests an important role for those two proteins in the induction of CYP $1 \mathrm{~A}$ by TCDD (Andreasen et al., 2002). However, the relation between CYP1A on one hand and the induction pathways AHR and ARNT2 and xenobiotics on the other hand remains complicated. Teraoka et al. (2003) showed that TCDD abnormalities can be prevented by CYP1A knockdown, while other research groups completely contradict this finding (Carney et al., 2004). Other organic toxins (e.g. tricyclic PAHs) must have an $A H R$ - and CYP1A-independent pathway of toxicity, since $A H R$ and $C Y P 1 A$ knockdown zebrafish embryos showed no decreased toxicity (Incardona et al., 2005). Surprisingly, the toxicity of tetracyclic PAH compounds is increased significantly by utilizing CYP1A morphants and reduced by AHR2 morphants. This indicates a protective influence of CYP1A towards such pollutants with an AHR2-dependent mechanism of toxicity (Billiard et al., 2006). Still, much debate is ongoing about the role of CYP1A and $A H R$ pathway in this observed toxicity. Furthermore, PXR may also be involved in $C Y P 1 A$ induction as a trend towards a reduced CYP1A expression is noted in zebrafish embryos when PXR is knocked down (Kubota et al., 2015).

Regardless of the underlying regulatory processes, it is clear that zebrafish CYP1A metabolizes environmental pollutants, but also many other human CYP substrates including ER, MR, 7-pentoxyresorufin (PR), and BR (Scornaienchi et al., 2010b). Furthermore, CYP1A in zebrafish, as in humans, is the sole CYP1 that shows 16- $\alpha$ hydroxylation activity of E2, despite the fact that the overall metabolism rate in zebrafish is much lower than that of human CYP1A1 (Scornaienchi et al., 2010a).

\section{CYP1B}

The only isoform of this subfamily in zebrafish is CYP1B1 (Godard et al., 2005), which is expressed in adults particularly in the eyes and the heart (Jönsson et al., 2007b). CYP1B1 expression showed a peak between 30 and 48 hpf and subsequent expression after hatching at $72 \mathrm{hpf}$ (Yin et al., 2008). Such a distinct spatio-temporal CYP1B1 pattern with considerable levels around $30 \mathrm{hpf}$ suggests an inherent role in eye development, as the retina begins to develop at this time (Easter and Nicola, 1996).

CYP1B1 modulation follows an AHR2 independ- ent pathway in the eye and the brain before hatching and an AHR2 dependent pathway in the branchial arches and the heart after hatching (Yin et al., 2008). However, the AHR2 independent pathway still needs AHR2 as a modulating factor. This dependency on AHR2 explains the absence of CYP1B1 expression in AHR2 knockdown embryos (Yin et al., 2008). Therefore, the induction by AHR agonists such as TCDD and 3, 3', 4, 4', 5-pentachlorobiphenyl (PCB126) is observed in both post-hatched embryos and adults (Jönsson et al., 2007b).

When compared to CYP1A, CYP1B1 showed much lower (Scornaienchi et al., 2010b) or even negligible (Stegeman et al., 2015) EROD activity in vitro. This is in accordance with data from Timme-Laragy et al. (2008), who showed that knocking down of CYP1B1 in zebrafish larvae had no effect on EROD activity. The latter confirms the contribution of other CYP1s to the observed high EROD activity in vivo.

The mechanisms of CYP1B1 action are similar across taxa. For example, the ratio of ER and E2 biotransformation by CYP1B1 to CYP1A in zebrafish is similar to the biotransformation by CYP1B1 to CYP1A1 in humans (Scornaienchi et al., 2010a). Furthermore, there is a high conservation of the E2 4-hydroxylation activation site in CYP1B1 proteins in both zebrafish and humans (Lewis et al., 1999). However, the ratio of E2 4-hydroxylation to E2 2-hydroxylation by zebrafish CYP1B1 is higher than this ratio by its human ortholog. This means that the main metabolite of E2 by zebrafish CYP1B1 is 4-hydroxy E2 while it is 2-hydroxy E2 by human CYP1B1 (Scornaienchi et al., 2010a). Additionally, zebrafish CYP1B1 can also metabolize dibenzylfluorescein (DBF), which is a CYP2C8, CYP2C9 and CYP3A4 substrate in human (Scornaienchi et al., 2010b). This indicates for distinctive metabolic characteristics of zebrafish CYP1B1 compared to human CYP1B1.

\section{CYP1Cs}

This subfamily is composed of two members in zebrafish, CYP1C1 and CYP1C2 (Godard et al., 2005), yet it has no counterpart in humans (Uno et al., 2012). The gene expression of CYP1Cs is higher than all other CYP1s at $80 \mathrm{hpf}$ (Jönsson et al., 2007b). The induction of these genes by AHR agonists reaches a peak at 4 days post fertilization (dpf) for CYP1C1, which remains at a high level during adulthood (Jönsson et al., 2007a, 2007b; Timme-Laragy et al., 2008; Wang et al., 2006). Moreover, the induction of CYP1Cs by AHR agonists shows a spatiotemporal trend. Hence, CYPICl is induced strongly by $3,3^{\prime}, 4,4^{\prime}, 5$-pentachlorobiphenyl in adults and embryos while it is negligible for CYP1C2 in adults 


\section{CYPs in zebrafish}

(Jönsson et al., 2007b). This induction is much higher in the mesenteric artery than in the liver (Bugiak and Weber, 2009). Both $C Y P 1 C 1$ and $C Y P 1 C 2$ have the highest basal expression in the heart and the eyes and the lowest in the ovaries. In particular, $C Y P 1 C 1$ and $C Y P 1 C 2$ also have high basal expressions in the gills and the kidneys, respectively (Jönsson et al., 2007b). This suggests an important biological and developmental role of this subfamily. Furthermore, vascular toxicity is reduced by silencing either $C Y P 1 C 1$ or $C Y P 1 C 2$, which indicates complementary functions of those two isoforms in embryos (Kubota et al., 2011).

Similar to CYP1A, CYP1C1 metabolizes E2 at the same rate (Scornaienchi et al., 2010a). Additionally, CYP1C1 and CYP1C2, at varying rates, can also metabolize numerous substrates and environmental pollutants. For instance, both subfamilies metabolize human CYP1 substrates, such as ER, MR and PR, and human CYP2 substrates, such as BR and the environmental contaminant BaP (Scornaienchi et al., 2010b; Stegeman et al., 2015). Interestingly, biotransformation of $\mathrm{BaP}$ by zebrafish CYP1Cs leads to metabolites that are similar to the ones formed by human CYP1A1. Furthermore, these CYP1C enzymes metabolize testosterone mainly to $6 \mathrm{~B}-\mathrm{OH}$-testosterone, which is a specific human CYP3A metabolite (Stegeman et al., 2015). This wide spectrum of substrates indicates an important role of this subfamily, which has no ortholog in man, in the biotransformation of endogenous substrates and detoxification of environmental pollutants in zebrafish.

\section{CYP1D}

In 2008, Goldstone et al. cloned a new CYP1 subfamily with one gene, namely $C Y P 1 D 1$, in the freshwater fish species medaka (Oryzias latipes). It was recently proven to be transcribed in many organs of adult zebrafish and in early stages of zebrafish development (Goldstone et al., 2009; Goldstone and Stegeman, 2008). A high level of CYP1D1 expression is detected in embryos at $9 \mathrm{hpf}$, but it reaches the highest level in adult zebrafish, more specifically in liver and brain (Goldstone et al., 2009). This gene has no functional protein in man (Nelson et al., 2004), though it does have in other mammals (Uno et al., 2011). Except for humans, the main function of this subfamily seems to be similar in mammals and other vertebrates (Goldstone et al., 2009).

Unlike other $C Y P 1 \mathrm{~s}, C Y P 1 D 1$ is not induced by $A H R$ agonists such as 6-formylindolo[3,2-b]carbazole (Jönsson et al., 2009) or by UV radiation exposure (Behrendt et al., 2010). It was not induced by PCB126 in killifish (Zanette et al., 2009), however, it was induced in
Cynomolgus monkey hepatocytes by omeprazole and rifampicin (Uno et al., 2011). Furthermore, CYP1D1 has low EROD and MROD activities, which are not affected by adding oxidative activators such as cytochrome b5 (Goldstone et al., 2009) and no activity towards other coumarin substrates (Stegeman et al., 2015). Subsequently, it does not metabolize E2 (Scornaienchi et al., 2010a), while there are low activities towards BaP (Scornaienchi et al., 2010b). The same is also observed with caffeine in some mammals rather than humans such as monkeys (Uno et al., 2011). Surprisingly, CYP1D1 metabolizes testosterone to $6 ß-\mathrm{OH}$-testosterone at much lower concentrations than CYP1Cs, but it forms two other undefined testosterone metabolites at higher concentrations than what is detected for CYP1Cs (Stegeman et al., 2015). These unique characteristics of CYP1D1 in zebrafish, and especially its low response towards different inducers and substrates, raise doubts about its role in the metabolism of xenobiotics.

\section{CYP2 FAMILY}

The CYP2 family comprises the largest CYP gene family in zebrafish, with 41 genes (Genome Reference Consortium, 2015). It is also the largest CYP gene family in humans containing 16 genes.

In both man and zebrafish, each $C Y P 2$ consists of nine exons, except for $C Y P 2 R$ and $C Y P 2 U$, which are composed of five exons. These exons show identical size in mammalian and ray-finned fishes, including zebrafish (Kirischian et al., 2011). In fish, CYP2s seem to be regulated via PXR and AHR (Mosadeghi et al., 2007; Yuan et al., 2013; Kubota et al., 2013, 2015). In contrast, several substrates tend to induce $C Y P 2 \mathrm{~s}$ in mammals, including humans, by the nuclear receptor CAR (Handschin and Meyer, 2003; Waxman, 1999).

\section{CYP2AA}

In zebrafish, the CYP2AA subfamily consists of 10 members (CYP2AA1-4, 6-9, 11, 12) (Genome Reference Consortium, 2015). Genes within this subfamily show $65-85 \%$ similarity to each other according to The Gene Wise algorithm, but no homology to mammalian CYP2s (Goldstone et al., 2010). The amino acids sequences of the predicted proteins of CYP2AA1 and 2AA2 are similar to the mammalian CYP2Bs, with a similarity of approximately 38-41\% (Kubota et al., 2013).

In adult zebrafish, CYP2AA1 is expressed in most tissues, with the intestine showing the highest expression levels. This gene has a variable response to PXR agonists. For instance, its mRNA levels increased after treatment 
with pregnenolone $16 \alpha$-carbonitrile $(\mathrm{PCN})$, but not with phenobarbital (PB), 1,4-bis [2-(3,5-dichloropyridyloxy)] benzene (TCPOBOP), or PCB126 (Kubota et al., 2013, 2015). In contrast, CYP2AA2 expression, which is highly abundant in the kidneys, was significantly upregulated by TCPOBOP and slightly by PB, but not by PCN or by PCB126 (Kubota et al., 2013). However, CYP2AA12 was induced by both PN and PCB126, and in both cases the induction could be partially suppressed by using morpholinos of PXR or AHR, respectively (Kubota et al., 2015). This suggests a role of the PXR pathway in the induction of both $C Y P 2 A A 1$ and $C Y P 2 A A 12$ but not $C Y P 2 A A 2$, and the AHR pathway in regulation of $C Y P 2 A A 12$ but not $C Y P 2 A A 1$ and $A A 2$.

In zebrafish embryos, CYP2AA7-9 and 2AA12 mRNA levels show peak expression at $24 \mathrm{hpf}$, followed by a decrease until $36 \mathrm{hpf}$, when it starts increasing slowly again. Moreover, CYP2AA4 is detected in unfertilized eggs (Goldstone et al., 2010). However, the functional meaning of these spatiotemporal patterns still needs to be further elucidated.

\section{CYP2AE}

This subfamily is unique to zebrafish and contains only one gene, i.e. CYP2AE1 (Genome Reference Consortium 2015). It shares no synteny with human CYPs or CYPs from other fish species (Kirischian et al., 2011) and its function is unknown yet.

\section{CYP2K}

This subfamily consists of 10 members (CYP2K6, 8, 16-22, 31) (Genome Reference Consortium 2015), which in general share synteny with the human $C Y P 2 W 1$ (Goldstone et al., 2010). The individual isoforms show different spatio-temporal patterns and a variable homology to other species. For example, CYP2K6 mRNA is only detected in liver and ovaries of adult zebrafish with a late onset of expression in the embryos. It is poorly expressed at 3 days post fertilization (dpf) and only reaches high levels at $5 \mathrm{dpf}$ in embryos/larvae. In contrast, CYP2K22 has earlier peaks of expression, i.e. at $3 \mathrm{hpf}$ and $48 \mathrm{hpf}$ (Goldstone et al., 2010). Furthermore, the amino acid sequence of zebrafish CYP2K6 is $63 \%$ identical to rainbow trout CYP2K 1 (Wang-Buhler et al., 2005).

Despite the orthological relationship between CYP2K1 in rainbow trout and CYP2K6 in zebrafish, their metabolic features are not completely the same. Both of them metabolize aflatoxin B1 (AFB1) to the carcinogenic exo-8, 9-AFB1 epoxide, whereas only rainbow trout $\mathrm{CYP} 2 \mathrm{~K} 1$ can metabolize lauric acid
(Wang-Buhler et al., 2005). Though CYP2K6 mRNA is not detected in embryos until $5 \mathrm{dpf}$ (Wang-Buhler et al., 2005), Weigt et al. (2011) showed that different AFB1-induced malformations were already observed at $1 \mathrm{dpf}$. It is unclear whether these malformations result from direct or indirect mutagenic effects of AFB1 itself or due to biotransformation of AFB1 by other CYPs than CYP2K6.

\section{CYP2AD, CYP2N, CYP2P, CYP2J, CYP2V}

The localization of the 11 genes (CYP2AD2,3,6, CYP2N13, CYP2P 6-10, CYP2J20, CYP2V1) in these subfamilies on chromosome 20 suggests a synteny with human CYP2J2 (Goldstone et al., 2010; Genome Reference Consortium, 2015), which is responsible for the biotransformation of arachidonic acid to cis-epoxyeicosatrienoic acids (Wang et al., 2005). In particular, the sequence of CYP2P6 putative protein is $50 \%$ similar to that of human CYP2J2, which makes it a good candidate for the biosynthesis of cis-epoxyeicosatrienoic acids (Wang et al., 2007b). CYP2P6 has a peak at $12 \mathrm{hpf}$, then decreasing to very low levels and increasing gradually again at $36 \mathrm{hpf}$. This isoform is expressed mainly in brain and gonads of adult zebrafish and slightly in liver, heart and kidneys. Its expression in adults fluctuates during the ovarian follicular maturation with high levels in parallel to the FSH and LH production stages. Moreover, it has high levels at the early oocyte developmental stages namely stage I and II, and reaches undetectable levels at stage III when the yolk proteins start to appear. Such expression patterns may be related to a female reproductive function of CYP2P6. Interestingly, silencing of the CYP2P6 gene has no effect on embryonic development, whereas the overexpression of this gene causes lethality, malformations in dorsal cords or the cardiovascular system and curved tails (Wang et al., 2007b).

For the rest of this group of genes, little is known about their function in zebrafish. CYP2P10, CYP2J20 and CYP2AD6 have peaks at $3 \mathrm{hpf}, \mathrm{CYP} 2 \mathrm{P} 7$ and CYP2V1 have increased levels between 6-12 hpf, whereas CYP2N13 has two peaks, i.e. at 3 and $48 \mathrm{hpf}$. The early detected expression suggests a role in the transition from maternally- to oocyte-derived transcript (Goldstone et al., 2010).

In other fish species, such as killifish (Fundulus heteroclitus), CYP2N1 and 2N2 were observed in the liver and the intestine, and the heart and the brain, respectively. Both of them show efficient epoxidation of arachidonic acid, as it is suggested for zebrafish CYP2P6, but also $\mathrm{N}$-demethylation of benzphetamine activities, and low O-dealkylase activities towards BR and PR (Oleksiak et al., 2000). On the other hand, CYP2P3 has a higher activ- 


\section{CYPs in zebrafish}

ity towards the previously mentioned resorufin substrates and benzphetamine than CYP2Ns (Oleksiak et al., 2003). Both CYP2Ns and CYP2P3 are suppressed by 12-O-tetradecanoylphorbol-13-acetate (Oleksiak et al., 2000, 2003). Hence, it is possible for the zebrafish homologous isoforms to have identical metabolic characters as those in killifish.

\section{CYP2R and CYP2U}

These two subfamilies exist in most of the studied vertebrate (Kirischian et al., 2011; Nelson et al., 2004) and invertebrate species (Zheng et al., 2013). In zebrafish, sequence analysis of $C Y P 2 R 1$ and $C Y P 2 U 1$ shows that both are classified as orthologs of human CYP genes, as they have very similar sequences and show synteny with their human counterparts (Goldstone et al., 2010).

Both isoforms play an important role in biotransformation, which is well documented in humans. Human CYP2R1 is involved in the metabolism of vitamin D into active metabolites (Cheng et al., 2003; Yasuda et al., 2013) and prostate carcinogenesis (Ellfolk et al., 2009), while CYP2U1 shares the activation of many fatty acids such as arachidonic acid (Strushkevich et al., 2008).

Interestingly, zebrafish embryos show higher CYP2R1 levels in low $\mathrm{Ca}^{+2}$ water, which also indicates a possible role of this isoform in vitamin D synthesis in zebrafish embryos (Lin et al., 2012).

\section{CYP2X}

Most ray-finned species express only one gene of this subfamily. In contrast, zebrafish has six genes namely, CYP 2 X6-9 and CYP $2 X 10$ that shows two similar copies (Genome Reference Consortium, 2015; Goldstone et al., 2010). These genes do not share synteny with any mammalian CYP (Goldstone et al., 2010; Nelson, 2009).

The function of this subfamily in zebrafish is unknown yet. However, some isoforms are well documented in other fish species, which suggests possible functions in zebrafish as well.

In channel catfish (Ictalurus punctatus), CYP2X1 has a broad spectrum distribution throughout the organism but low biotransformation activities and induction responses. This isoform is detected in all tissues but especially in the liver and the gills. However, it has a very low $\mathrm{N}$-demethylation activity towards aminopyrine and benzphetamine when compared to other CYP2 subfamilies in fish. Furthermore, it has no activity towards alkoxyresorufin substrates and p-nitrophenol, which are usually general CYP2 substrates. Except the significant suppression of mRNA expression in the kidneys, no changes were observed with other CYP inducers and suppres- sors such as ethanol, clofibric acid, rifampin and pyridine (Mosadeghi et al., 2007).

On the other hand, the expression of CYP2X10 in goldfish (Carassius auratus) increased significantly after exposure to environmental pollutants, which indicates a possible role of this subfamily in biotransformation of xenobiotics (Wang et al., 2007a).

\section{CYP2Y}

CYP2Y3 is the only member of this subfamily in zebrafish (Genome Reference Consortium, 2015). It shares synteny with human $C Y P 2 A 6, C Y P 2 A 13, C Y P 2 B 6$, $C Y P 2 F 1$, and CYP2S1 (Goldstone et al., 2010). Zebrafish CYP2Y3 is identical to the CYP2Y3 found in Atlantic cod (Olsvik et al., 2009). Though little is known about the activity of this subfamily in zebrafish, several studies detected the effects of different toxins on CYP2Y3 expression in other fish species. Many differences were observed in the response to different substrates, inducers and suppressors between this subfamily in fish and its mammalian homologs. For instance, nonylphenol (NP), which is a PXR-mediated CYP-inducer in mice (Mota et al., 2011), and bisphenol A (BPA), which is a suppressor for some rat CYPs (Pfeiffer and Metzler, 2004), cause a reduction in CYP2Y3 mRNAs levels in Atlantic cod (Olsvik et al., 2009). Another example for differential responses between species is 2,2',4,4'tetrabromodiphenyl ether PBDE-47, an environmental toxin. It induces human CYP2B6 by PXR and CAR pathways (Sueyoshi et al., 2014) and is also significantly metabolized by human CYP2B6 (Feo et al., 2013). However, this compound does not affect the expression of CYP2Y3 in Atlantic cod (Olsvik et al., 2009), despite the synteny between CYP2Y3 and human CYP2B6 (Goldstone et al., 2010). This suggests that different regulatory mechanisms may be involved for this subfamily in fish species rather than PXR and CAR induction pathways. Yuan et al. (2013) showed some evidence of Chinese rare minnow CYP2Y3 induction, which shares $75 \%$ pair-wise identity with zebrafish CYP2Y3 putative protein, by BaP, which is an AHR agonist. This suggests a role for AHR-linked induction of $C Y P 2 Y 3$, but this needs further investigation.

\section{CYP3 FAMILY}

In zebrafish, this family consists out of only five members, i.e. CYP3A65 and CYP3C1-4 (Goldstone et al., 2010). Numerous human CYP3 substrates are metabolized by zebrafish, though differences in metabolization profiles are reported, e.g. for testosterone (Chng et al., 2012). The metabolization profile of testosterone is also 
different from zebrafish adult compared to larvae (Chng et al., 2012). This may be due to the variable expression of CYPs throughout the development. In general, in zebrafish, only $25 \%$ of CYPs has an elevated trend during the first $2 \mathrm{dpf}$, while the rest shows various temporal patterns (Goldstone et al., 2010).

Similar to human CYP3As, zebrafish CYP3As are involved in the biotransformation of drugs such as emodin, an herbal compound for diverse human therapeutic indications. However, toxic metabolites of emodin are reported in zebrafish, whereas this compound is safe in man (He et al., 2012). Additionally, Alderton et al. (2010) showed that for midazolam, which is a well-known human CYP3A tool compound, no metabolites could be detected in zebrafish larvae (Alderton et al., 2010). This makes CYP3 activity in zebrafish still a controversial issue.

\section{CYP3A}

CYP3A65 is the only known isoform of this subfamily in zebrafish. It is detected at low levels at $24 \mathrm{hpf}$, with an increasing trend after $72 \mathrm{hpf}$ typically in the foregut, and later in the liver and the intestine of adults. Other organs such as the brain, the gills, and the eyes express low levels of CYP3A65 (Tseng et al., 2005).

The nuclear receptor PXR is an important transcriptional pathway of CYP3As in human beings and mammals in general, which can be induced by several xenobiotics such as rifampicin, clotrimazole and nifedipine in man and dexamethasone and pregnenolone 16a-carboninitrile (PCN) in mice (Coumoul et al., 2002; Liddle and Goodwin, 2002; Moore et al., 2002; Guengerich, 1999). In zebrafish larvae, CYP3A65 is also induced by pregnenolone $(\mathrm{PN})$, rifampicin and dexamethasone (Tseng et al., 2005; Kubota et al., 2015). Clotrimazole and nifedipine show no effect on CYP3A65 mRNA levels in adult liver, whereas PCN increases significantly these levels (Bresolin et al., 2005). However, both basal and TCDDinduced levels of CYP3A65 mRNA in zebrafish were negligible in AHR2 morpholino embryos (Tseng et al., 2005). In contrast, Kubota et al. (2015) showed elevated levels of CYP3A65 mRNA after exposure to PCB126 and these induced levels were decreased in AHR knockdown embryos. These findings indicate that CYP3A65 expression is regulated by AHR2 and PXR pathways (Chang et al., 2013; Kubota et al., 2015).

Similar to human CYP3As, zebrafish CYP3A65 plays an important role in the detoxification of environmental hepatotoxins and in drug metabolism such as microcystin and testosterone (Chng et al., 2012; Li et al., 2013). Also 2-hydroxy E2 is the main metabolite of E2 via CYP3A in both zebrafish and humans (Scornaienchi et al., 2010a).
Still differences are reported. For instance, BFC, which is a specific human CYP3A substrate, is biotransformed more efficiently in zebrafish by CYP1A rather than CYP3A65 (Scornaienchi et al., 2010b).

\section{CYP3C}

This subfamily consists of four genes (CYP3C1-4) (Goldstone et al., 2010) and shares synteny with human CYP3A3, $3 A 4$ and $3 A 7$ (Qiu et al., 2008). Corley-Smith et al. (2006) showed CYP3C1 mRNA levels in embryos at $12 \mathrm{hpf}$, which are distributed in the whole embryo until 48 hpf. At 120 hpf, CYP3C1 concentrates in the brain and appears in the pharynx and the gastrointestinal system. However, in adults, CYP3C1 mRNA levels are absent in the brain, low in the heart and the eyes and high in the liver, the intestines, and the ovaries (Corley-Smith et al., 2006).

In zebrafish embryos, Corley-Smith et al. (2006) suggested that CYP3C1 modulation is independent from the PXR and the AHR pathways as its mRNA levels were not affected by TCDD, dexamethasone or rifampicin (Corley-Smith et al., 2006). In contrast, Kubota et al. (2015) showed a possible role of PXR and AHR pathways in CYP3C1 regulation as it was induced by both $\mathrm{PN}$ and PCB126, and this induction was partially reversed when using PXR and AHR morpholinos, respectively (Kubota et al., 2015). This discrepancy in results on CYP3C1 regulation may be due to the difference in substrates that were used by both groups. Additionally, Corley-Smith et al. (2006) applied a continuous exposure of fertilized eggs until $120 \mathrm{hpf}$ at relatively high substrate concentrations, whereas Kubota et al. (2015) exposed different developmental stages for only $24 \mathrm{hr}$ at relatively low substrate concentrations. The latter exposure window was specifically chosen as preliminary experiments had shown a lower induction of CYPs and receptors when $48 \mathrm{hpf}$ embryos were exposed to $\mathrm{PN}$ for $48 \mathrm{hr}$ instead of $24 \mathrm{hr}$. Interestingly, knockdown of Krüppel-like factor 6 caused a decrease in CYP3C1 expression in $96 \mathrm{hpf}$ zebrafish embryos (Zhao et al., 2010). However, as these morphants also have small livers, the low expression levels of CYP3C1 may be rather due to the low number of hepatocytes in these embryos than caused by a direct effect of Krüppel-like factor 6 on CYP3C1.

\section{Conclusion}

Zebrafish CYPs, as the most important enzymes in biotransformation, show a strong evolutionary and orthological relationship to humans. Regarding CYP activity, several studies revealed shared metabolic characteristics with humans. Yet, important differences in the metabolite 
CYPs in zebrafish

profile of many substrates, gene expression inhibition and induction have also been reported.

Taken altogether, these data indicate that a distinct paucity in information regarding xenobiotic CYP activity within family 1-3 exists. Uncovering these elusive mechanisms will aid in enhancing developmental toxicity tests, which contain contradicting information regarding CYP activity and function. With increasing knowledge on the biotransformation processes in zebrafish at different developmental stages compared to humans, the zebrafish can be further validated and strengthened as a vertebrate model in drug discovery and toxicology.

Conflict of interest---- The authors declare that there is no conflict of interest.

\section{REFERENCES}

Alderton, W., Berghmans, S., Butler, P., Chassaing, H., Fleming, A., Golder, Z., Richards, F. and Gardner, I. (2010): Accumulation and metabolism of drugs and CYP probe substrates in zebrafish larvae. Xenobiotica, 40, 547-557.

Andreasen, E.A., Spitsbergen, J.M., Tanguay, R.L., Stegeman, J.J., Heideman, W. and Peterson, R.E. (2002): Tissue-specific expression of AHR2, ARNT2, and CYP1A in zebrafish embryos and larvae: effects of developmental stage and 2,3,7,8-tetrachlorodibenzo-p-dioxin exposure. Toxicol. Sci., 68, 403-419.

Behrendt, L., Jönsson, M.E., Goldstone, J.V. and Stegeman, J.J. (2010): Induction of cytochrome P450 1 genes and stress response genes in developing zebrafish exposed to ultraviolet radiation. Aquat. Toxicol., 98, 74-82.

Beker van Woudenberg, A., Wolterbeek, A., Te Brake, L., Snel, C., Menke, A., Rubingh, C., de Groot, D. and Kroese, D. (2013): A category approach to predicting the developmental (neuro) toxicity of organotin compounds: the value of the zebrafish (Danio rerio) embryotoxicity test (ZET). Reprod. Toxicol., 41, 35-44.

Billiard, S.M., Timme-Laragy, A.R., Wassenberg, D.M., Cockman, C. and Di Giulio, R.T. (2006): The role of the aryl hydrocarbon receptor pathway in mediating synergistic developmental toxicity of polycyclic aromatic hydrocarbons to zebrafish. Toxicol. Sci., 92, 526-536.

Brannen, K.C., Panzica-Kelly, J.M., Danberry, T.L. and Augustine-Rauch, K.A. (2010): Development of a zebrafish embryo teratogenicity assay and quantitative prediction model. Birth Defects Res. B Dev. Reprod. Toxicol., 89, 66-77.

Bresolin, T., de Freitas, R.M. and Celso Dias, B.A. (2005): Expression of PXR, CYP3A and MDR1 genes in liver of zebrafish. Comp. Biochem. Physiol. C. Toxicol. Pharmacol., 140, 403-407.

Brown, C.M., Reisfeld, B. and Mayeno, A.N. (2008): Cytochromes P450: a structure-based summary of biotransformations using representative substrates. Drug Metab. Rev., 40, 1-100.

Bugiak, B. and Weber, L.P. (2009): Hepatic and vascular mRNA expression in adult zebrafish (Danio rerio) following exposure to benzo-a-pyrene and 2,3,7,8-tetrachlorodibenzo-p-dioxin. Aquat. Toxicol., 95, 299-306.

Carney, S.A., Peterson, R.E. and Heideman, W. (2004): 2,3,7,8Tetrachlorodibenzo-p-dioxin activation of the aryl hydrocarbon receptor/aryl hydrocarbon receptor nuclear translocator pathway causes developmental toxicity through a CYP1A-independent mechanism in zebrafish. Mol. Pharmacol., 66, 512-521.

Chang, C.T., Chung, H.Y., Su, H.T., Tseng, H.P., Tzou, W.S. and Hu, C.H. (2013): Regulation of zebrafish CYP3A65 transcription by AHR2. Toxicol. Appl. Pharmacol., 270, 174-184.

Cheng, J.B., Motola, D.L., Mangelsdorf, D.J. and Russell, D.W. (2003): De-orphanization of cytochrome P450 2R1: a microsomal vitamin D 25-hydroxilase. J. Biol. Chem., 278, 38084-38093.

Chng, H.T., Ho, H.K., Yap, C.W., Lam, S.H. and Chan, E.C. (2012): An investigation of the bioactivation potential and metabolism profile of Zebrafish versus human. J. Biomol. Screen, 17, 974986.

Corley-Smith, G.E., Su, H.T., Wang-Buhler, J.L., Tseng, H.P., Hu, C.H., Hoang, T., Chung, W.G. and Buhler, D.R. (2006): CYP3C1, the first member of a new cytochrome P450 subfamily found in zebrafish (Danio rerio). Biochem. Biophys. Res. Commun., 340, 1039-1046.

Coumoul, X., Diry, M. and Barouki, R. (2002): PXR-dependent induction of human CYP3A4 gene expression by organochlorine pesticides. Biochem. Pharmacol., 64, 1513-1519.

Crespi, C.L. and Stresser, D.M. (2000): Fluorometric screening for metabolism-based drug--drug interactions. J. Pharmacol. Toxicol. Methods, 44, 325-331.

Denison, M.S. and Nagy, S.R. (2003): Activation of the aryl hydrocarbon receptor by structurally diverse exogenous and endogenous chemicals. Annu. Rev. Pharmacol. Toxicol., 43, 309-334.

Easter, S.S.Jr. and Nicola, G.N. (1996): The development of vision in the zebrafish (Danio rerio). Dev. Biol., 180, 646-663.

Ekins, S., Reschly, E.J., Hagey, L.R. and Krasowski, M.D. (2008): Evolution of pharmacologic specificity in the pregnane $\mathrm{X}$ receptor. BMC. Evol. Biol., 8, 103.

Ellfolk, M., Norlin, M., Gyllensten, K. and Wikvall, K. (2009): Regulation of human vitamin $\mathrm{D}(3)$ 25-hydroxylases in dermal fibroblasts and prostate cancer LNCaP cells. Mol. Pharmacol., 75, 1392-1399.

Feo, M.L., Gross, M.S., McGarrigle, B.P., Eljarrat, E., Barceló, D., Aga, D.S. and Olson, J.R. (2013): Biotransformation of BDE47 to potentially toxic metabolites is predominantly mediated by human CYP2B6. Environ. Health Perspect., 121, 440-446.

Franklin, M.R. (1977): Toxic effects. Science, 198, 1029-1030.

Genome Reference Consortium (2015): GRCz10.

Godard, C.A., Goldstone, J.V., Said, M.R., Dickerson, R.L., Woodin, B.R. and Stegeman, J.J. (2005): The new vertebrate CYP1C family: cloning of new subfamily members and phylogenetic analysis. Biochem. Biophys. Res. Commun., 331, 10161024.

Goldstone, J.V., Jönsson, M.E., Behrendt, L., Woodin, B.R., Jenny, M.J., Nelson, D.R. and Stegeman, J.J. (2009): Cytochrome P450 1D1: a novel CYP1A-related gene that is not transcriptionally activated by PCB126 or TCDD. Arch. Biochem. Biophys., 482, 7-16.

Goldstone, J.V., McArthur, A.G., Kubota, A., Zanette, J., Parente, T., Jönsson, M.E., Nelson, D.R. and Stegeman, J.J. (2010): Identification and developmental expression of the full complement of Cytochrome P450 genes in Zebrafish. BMC. Genomics, 11, 643.

Goldstone, J.V. and Stegeman, J.J. (2008): Gene structure of the novel cytochrome P4501D1 genes in stickleback (Gasterosteus aculeatus) and medaka (Oryzias latipes). Mar. Environ. Res., 66, 19-20.

Guengerich, F.P. (1999): Cytochrome P-450 3A4: regulation and role in drug metabolism. Annu. Rev. Pharmacol. Toxicol., 39, 
M. Saad et al.

$1-17$.

Guengerich, F.P. (2008): Cytochrome P450 and chemical toxicology. Chem. Res. Toxicol., 21, 70-83.

Hahn, M.E. (2002): Aryl hydrocarbon receptors: diversity and evolution. Chem. Biol. Interact., 141, 131-160.

Handschin, C. and Meyer, U.A. (2003): Induction of drug metabolism: the role of nuclear receptors. Pharmacol. Rev., 55, 649673.

He, Q., Liu, K., Wang, S., Hou, H., Yuan, Y. and Wang, X. (2012): Toxicity induced by emodin on zebrafish embryos. Drug Chem. Toxicol., 35, 149-154.

Henry, T.R., Spitsbergen, J.M., Hornung, M.W., Abnet, C.C. and Peterson, R.E. (1997): Early life stage toxicity of 2,3,7,8-tetrachlorodibenzo-p-dioxin in zebrafish (Danio rerio). Toxicol. Appl. Pharmacol., 142, 56-68.

Incardona, J.P., Carls, M.G., Teraoka, H., Sloan, C.A., Collier, T.K. and Scholz, N.L. (2005): Aryl hydrocarbon receptor-independent toxicity of weathered crude oil during fish development. Environ. Health Perspect., 113, 1755-1762.

Jönsson, M.E., Franks, D.G., Woodin, B.R., Jenny, M.J., Garrick, R.A., Behrendt, L., Hahn, M.E. and Stegeman, J.J. (2009): The tryptophan photoproduct 6-formylindolo[3,2-b]carbazole (FICZ) binds multiple AHRs and induces multiple CYP1 genes via AHR2 in zebrafish. Chem. Biol. Interact, 181, 447-454.

Jönsson, M.E., Jenny, M.J., Woodin, B.R., Hahn, M.E. and Stegeman, J.J. (2007a): Role of AHR2 in the expression of novel cytochrome P450 1 family genes, cell cycle genes, and morphological defects in developing zebra fish exposed to $3,3^{\prime}, 4,4^{\prime}, 5-$ pentachlorobiphenyl or 2,3,7,8-tetrachlorodibenzo-p-dioxin. Toxicol. Sci., 100, 180-193.

Jönsson, M.E., Orrego, R., Woodin, B.R., Goldstone, J.V. and Stegeman, J.J. (2007b): Basal and 3,3',4,4',5-pentachlorobiphenyl-induced expression of cytochrome P450 1A, 1B and 1C genes in zebrafish. Toxicol. Appl. Pharmacol., 221, 29-41.

Karchner, S.I., Franks, D.G. and Hahn, M.E. (2005): AHR1B, a new functional aryl hydrocarbon receptor in zebrafish: tandem arrangement of ahr1b and ahr2 genes. Biochem. J., 392, 153161.

Kirischian, N., McArthur, A.G., Jesuthasan, C., Krattenmacher, B. and Wilson, J.Y. (2011): Phylogenetic and functional analysis of the vertebrate cytochrome p450 2 family. J. Mol. Evol., 72, 56-71.

Kubota, A., Bainy, A.C., Woodin, B.R., Goldstone, J.V. and Stegeman, J.J. (2013): The cytochrome P450 2AA gene cluster in zebrafish (Danio rerio): expression of CYP2AA1 and CYP2AA2 and response to phenobarbital-type inducers. Toxicol. Appl. Pharmacol., 272, 172-179.

Kubota, A., Goldstone, J.V., Lemaire, B., Takata, M., Woodin, B.R. and Stegeman, J.J. (2015): Role of pregnane X receptor and aryl hydrocarbon receptor in transcriptional regulation of pxr, CYP2, and CYP3 genes in developing zebrafish. Toxicol. Sci., 143, 398-407.

Kubota, A., Stegeman, J.J., Woodin, B.R., Iwanaga, T., Harano, R., Peterson, R.E., Hiraga, T. and Teraoka, H. (2011): Role of zebrafish cytochrome P450 CYP1C genes in the reduced mesencephalic vein blood flow caused by activation of AHR2. Toxicol. Appl. Pharmacol., 253, 244-252.

Lewis, D.F., Lake, B.G., George, S.G., Dickins, M., Eddershaw, P.J., Tarbit, M.H., Beresford, A.P., Goldfarb, P.S. and Guengerich, F.P. (1999): Molecular modelling of CYP1 family enzymes CYP1A1, CYP1A2, CYP1A6 and CYP1B1 based on sequence homology with CYP102. Toxicology, 139, 53-79.
Li, X., Ma, J., Fang, Q. and Li, Y. (2013): Transcription alterations of microRNAs, cytochrome P4501A1 and 3A65, and AhR and PXR in the liver of zebrafish exposed to crude microcystins. Toxicon, 73, 17-22

Liddle, C. and Goodwin, B. (2002): Regulation of hepatic drug metabolism: role of the nuclear receptors PXR and CAR. Semin. Liver Dis., 22, 115-122.

Lin, C.H., Su, C.H., Tseng, D.Y., Ding, F.C. and Hwang, P.P. (2012): Action of vitamin D and the receptor, VDRa, in calcium handling in zebrafish (Danio rerio). PLoS One, 7, e45650.

Mattingly, C.J. and Toscano, W.A. (2001): Posttranscriptional silencing of cytochrome P4501A1 (CYP1A1) during zebrafish (Danio rerio) development. Dev. Dyn., 222, 645-654.

Merchant, M., Wang, X., Kamps, C., Rosengren, R., Morrison, V. and Safe, S. (1992): Mechanism of benzo[a]pyrene-induced Cypla-1 gene expression in mouse Hepa 1c1c7 cells: role of the nuclear $6 \mathrm{~s}$ and $4 \mathrm{~s}$ proteins. Arch. Biochem. Biophys., 292, 250257.

Miranda, C.L., Henderson, M.C. and Buhler, D.R. (1998): Evaluation of chemicals as inhibitors of trout cytochrome P450s. Toxicol. Appl. Pharmacol., 148, 237-244.

Moore, L.B., Maglich, J.M., McKee, D.D., Wisely, B., Willson, T.M., Kliewer, S.A., Lambert, M.H. and Moore, J.T. (2002): Pregnane $\mathrm{X}$ receptor (PXR), constitutive androstane receptor (CAR), and benzoate $\mathrm{X}$ receptor (BXR) define three pharmacologically distinct classes of nuclear receptors. Mol. Endocrinol., 16, 977-986.

Mosadeghi, S., Furnes, B., Matsuo, A.Y. and Schlenk, D. (2007): Expression and characterization of cytochrome P450 2X1 in channel catfish (Ictalurus punctatus). Biochim. Biophys. Acta, 1770, 1045-1052.

Mota, L.C., Barfield, C., Hernandez, J.P. and Baldwin, W.S. (2011): Nonylphenol-mediated CYP induction is PXR-dependent: The use of humanized mice and human hepatocytes suggests that hPXR is less sensitive than mouse PXR to nonylphenol treatment. Toxicol. Appl. Pharmacol., 252, 259-267.

Nelson, D.R. (2009): The cytochrome p450 homepage. Hum. Genomics, 4, 59-65.

Nelson, D.R., Zeldin, D.C., Hoffman, S.M., Maltais, L.J., Wain, H.M. and Nebert, D.W. (2004): Comparison of cytochrome P450 (CYP) genes from the mouse and human genomes, including nomenclature recommendations for genes, pseudogenes and alternative-splice variants. Pharmacogenetics, 14, 1-18.

Niwa, T., Shiraga, T., Yamasaki, S., Ishibashi, K., Ohno, Y. and Kagayama, A. (2003): In vitro activation of 7-benzyloxyresorufin O-debenzylation and nifedipine oxidation in human liver microsomes. Xenobiotica, 33, 717-729.

Oleksiak, M.F., Wu, S., Parker, C., Karchner, S.I., Stegeman, J.J. and Zeldin, D.C. (2000): Identification, functional characterization, and regulation of a new cytochrome P450 subfamily, the CYP2Ns. J. Biol. Chem., 275, 2312-2321.

Oleksiak, M.F., Wu, S., Parker, C., Qu, W., Cox, R., Zeldin, D.C. and Stegeman, J.J. (2003): Identification and regulation of a new vertebrate cytochrome P450 subfamily, the CYP2Ps, and functional characterization of $\mathrm{CYP} 2 \mathrm{P} 3$, a conserved arachidonic acid epoxygenase/19-hydroxylase. Arch. Biochem. Biophys., 411, 223-234.

Olsvik, P.A., Lie, K.K., Sturve, J., Hasselberg, L. and Andersen, O.K. (2009): Transcriptional effects of nonylphenol, bisphenol A and PBDE-47 in liver of juvenile Atlantic cod (Gadus morhua). Chemosphere, 75, 360-367.

Otte, J.C., Schmidt, A.D., Hollert, H. and Braunbeck, T. (2010): 
CYPs in zebrafish

Spatio-temporal development of CYP1 activity in early life-stages of zebrafish (Danio rerio). Aquat. Toxicol., 100, 38-50.

Pfeiffer, E. and Metzler, M. (2004): Effect of bisphenol A on drug metabolising enzymes in rat hepatic microsomes and precisioncut rat liver slices. Arch. Toxicol., 78, 369-377.

Qiu, H., Taudien, S., Herlyn, H., Schmitz, J., Zhou, Y., Chen, G., Roberto, R., Rocchi, M., Platzer, M. and Wojnowski, L. (2008): CYP3 phylogenomics: evidence for positive selection of CYP3A4 and CYP3A7. Pharmacogenet. Genomics, 18, 53-66.

Reschly, E.J. and Krasowski, M.D. (2006): Evolution and function of the NR1I nuclear hormone receptor subfamily (VDR, PXR, and CAR) with respect to metabolism of xenobiotics and endogenous compounds. Curr. Drug Metab., 7, 349-365.

Scornaienchi, M.L., Thornton, C., Willett, K.L. and Wilson, J.Y. (2010a): Cytochrome P450-mediated 17beta-estradiol metabolism in zebrafish (Danio rerio). J. Endocrinol., 206, 317-325.

Scornaienchi, M.L., Thornton, C., Willett, K.L. and Wilson, J.Y. (2010b): Functional differences in the cytochrome P450 1 family enzymes from zebrafish (Danio rerio) using heterologously expressed proteins. Arch. Biochem. Biophys., 502, 17-22.

Shimada, T., Yamazaki, H., Mimura, M., Wakamiya, N., Ueng, Y.F., Guengerich, F.P. and Inui, Y. (1996): Characterization of microsomal cytochrome P450 enzymes involved in the oxidation of xenobiotic chemicals in human fetal liver and adult lungs. Drug Metab. Dispos., 24, 515-522.

Stegeman, J.J., Behrendt, L., Woodin, B.R., Kubota, A., Lemaire, B., Pompon, D., Goldstone, J.V. and Urban, P. (2015): Functional characterization of zebrafish cytochrome P450 1 family proteins expressed in yeast. Biochim. Biophys. Acta, 1850, 2340-2352.

Strushkevich, N., Usanov, S.A., Plotnikov, A.N., Jones, G. and Park, H.W. (2008): Structural analysis of CYP2R1 in complex with vitamin D3. J. Mol. Biol., 380, 95-106.

Sueyoshi, T., Li, L., Wang, H., Moore, R., Kodavanti, P.R., Lehmler, H.J., Negishi, M. and Birnbaum, L.S. (2014): Flame retardant BDE-47 effectively activates nuclear receptor CAR in human primary hepatocytes. Toxicol. Sci., 137, 292-302.

Teraoka, H., Dong, W., Tsujimoto, Y., Iwasa, H., Endoh, D., Ueno, N., Stegeman, J.J., Peterson, R.E. and Hiraga, T. (2003): Induction of cytochrome P450 1A is required for circulation failure and edema by 2,3,7,8-tetrachlorodibenzo-p-dioxin in zebrafish. Biochem. Biophys. Res. Commun., 304, 223-228.

Timme-Laragy, A.R., Noyes, P.D., Buhler, D.R. and Di Giulio, R.T. (2008): CYP1B1 knockdown does not alter synergistic developmental toxicity of polycyclic aromatic hydrocarbons in zebrafish (Danio rerio). Mar. Environ. Res., 66, 85-87.

Tseng, H.P., Hseu, T.H., Buhler, D.R., Wang, W.D. and Hu, C.H. (2005): Constitutive and xenobiotics-induced expression of a novel CYP3A gene from zebrafish larva. Toxicol. Appl. Pharmacol., 205, 247-258.

Uno, T., Ishizuka, M. and Itakura, T. (2012): Cytochrome P450 (CYP) in fish. Environ. Toxicol. Pharmacol., 34, 1-13.

Uno, Y., Uehara, S., Murayama, N. and Yamazaki, H. (2011): CYP1D1, pseudogenized in human, is expressed and encodes a functional drug-metabolizing enzyme in cynomolgus monkey. Biochem. Pharmacol., 81, 442-450.

Wang-Buhler, J.L., Lee, S.J., Chung, W.G., Stevens, J.F., Tseng, H.P., Hseu, T.H., Hu, C.H., Westerfield, M., Yang, Y.H., Miranda, C.L. and Buhler, D.R. (2005): CYP2K6 from zebrafish (Danio rerio): cloning, mapping, developmental/ tissue expression, and aflatoxin $\mathrm{B} 1$ activation by baculovirus expressed enzyme. Comp Biochem. Physiol C. Toxicol. Pharmacol., 140, 207-219.

Wang, J., Wei, Y., Li, X., Xu, M. and Dai, J. (2007a): Identification of differentially expressed genes from contaminant and thermal exposed goldfish Carassius auratus in Gaobeidian Lake in Beijing, China. Ecotoxicology, 16, 525-532.

Wang, L., Scheffler, B.E. and Willett, K.L. (2006): CYP1C1 messenger RNA expression is inducible by benzo[a]pyrene in Fundulus heteroclitus embryos and adults. Toxicol. Sci., 93, 331340 .

Wang, L., Yao, J., Chen, L., Chen, J., Xue, J. and Jia, W. (2007b): Expression and possible functional roles of cytochromes P450 2J1 (zfCyp 2J1) in zebrafish. Biochem. Biophys. Res. Commun., 352, 850-855.

Wang, Y., Wei, X., Xiao, X., Hui, R., Card, J.W., Carey, M.A., Wang, D.W. and Zeldin, D.C. (2005): Arachidonic acid epoxygenase metabolites stimulate endothelial cell growth and angiogenesis via mitogen-activated protein kinase and phosphatidylinositol 3-kinase/Akt signaling pathways. J. Pharmacol. Exp. Ther., 314, 522-532.

Waxman, D.J. (1999): P450 gene induction by structurally diverse xenochemicals: central role of nuclear receptors CAR, PXR, and PPAR. Arch. Biochem. Biophys., 369, 11-23.

Weigt, S., Huebler, N., Strecker, R., Braunbeck, T. and Broschard, T.H. (2011): Zebrafish (Danio rerio) embryos as a model for testing proteratogens. Toxicology, 281, 25-36.

Weigt, S., Huebler, N., Strecker, R., Braunbeck, T. and Broschard, T.H. (2012): Developmental effects of coumarin and the anticoagulant coumarin derivative warfarin on zebrafish (Danio rerio) embryos. Reprod. Toxicol., 33, 133-141.

Yasuda, K., Endo, M., Ikushiro, S., Kamakura, M., Ohta, M. and Sakaki, T. (2013): UV-dependent production of 25-hydroxyvitamin D2 in the recombinant yeast cells expressing human CYP2R1. Biochem. Biophys. Res. Commun., 434, 311-315.

Yin, H.C., Tseng, H.P., Chung, H.Y., Ko, C.Y., Tzou, W.S., Buhler, D.R. and Hu, C.H. (2008): Influence of TCDD on zebrafish CYP1B1 transcription during development. Toxicol. Sci., 103, 158-168.

Yuan, L., Lv, B., Zha, J., Wang, Z., Wang, W., Li, W. and Zhu, L. (2013): New cytochrome P450 1B1, 1C1, 2Aa, 2Y3, and 2K genes from Chinese rare minnow (Gobiocypris rarus): Molecular characterization, basal expression and response of rare minnow CYP1s and CYP2s mRNA exposed to the AHR agonist benzo[a]pyrene. Chemosphere, 93, 209-216.

Zanette, J., Jenny, M.J., Goldstone, J.V., Woodin, B.R., Watka, L.A., Bainy, A.C. and Stegeman, J.J. (2009): New cytochrome P450 $1 \mathrm{~B} 1,1 \mathrm{C} 2$ and 1D1 genes in the killifish Fundulus heteroclitus: Basal expression and response of five killifish CYP1s to the AHR agonist PCB126. Aquat. Toxicol., 93, 234-243.

Zhao, X., Monson, C., Gao, C., Gouon-Evans, V., Matsumoto, N., Sadler, K.C. and Friedman, S.L. (2010): Klf6/copeb is required for hepatic outgrowth in zebrafish and for hepatocyte specification in mouse ES cells. Dev. Biol., 344, 79-93.

Zheng, S., Chen, B., Qiu, X., Lin, K. and Yu, X. (2013): Three novel cytochrome P450 genes identified in the marine polychaete Perinereis nuntia and their transcriptional response to xenobiotics. Aquat. Toxicol., 134-135, 11-22. 